\title{
SIMULATION MODEL EVOLUTION A STRATEGIC TOOL FOR MODEL PLANNING
}

\author{
Todd Hunter \\ Eaton-Kenway, Inc. \\ 515 East 100 South \\ Salt Lake City, Utah 84102, U.S.A.
}

\begin{abstract}
Most simulation models evolve over time through a number of different metamorphic states. These states are typical to most simulation efforts within a specific simulation category (ie. health care, military, material handling etc.). Using a cursory SWOT (strengths, weaknesses, opportunities, threats) analysis approach, this paper seeks to define the states as they occur in a typical automated material handling simulation effort. The definition of each state includes an applicable SWOT analysis. Understanding these states enables simulation professionals to better develop meaningful strategies for future simulation, and aids in the creation of economies of scale.
\end{abstract}

\section{INTRODUCTION}

This paper presents the SWOT analysis for each of three distinct phases of a material handling system. The phases are described as follows:

Design Phase. During this phase, the system is conceptualized and designed to meet the material handling needs as described by the end user.

Construction Phase. During this phase, the system is constructed as designed.

Service Phase. During this phase, the system is fully operational and in use by the end user.

As the system progresses through each of these phases, the simulation model, as well as the effect that the results can have on the system have specific strengths, weaknesses, opportunities and threats. The strengths and weaknesses pertain to the model itself, while the opportunities and threats pertain more to the effect that the results can have on the material handling system.

\section{ANALYSIS}

The following narrative discusses the analysis of each phase.

\subsection{Design Phase}

During the design phase of the system we are assuming complete freedom to design the system as will best meet the needs of the end user.

\subsubsection{Strengths}

The strengths of the model during this phase relate to its ability to influence the design of the system. One of the primary strengths is the ability of the model results to force closure between the needs expressed by the end user, and the design created by the material handling system supplier. This closure is necessary to eliminate the difference between what the end user first envisions the system to be, and what is designed by the material handling suppliers. The closure is vital to the success of the system and is often reached after several significant revisions to the model.

Another significant strength is the ability of the model to educate the user about the system. This is especially applicable for simulation efforts that involve the use of graphics. During this phase, the prospective system user can get a good idea of just how his system will look and feel. This is often helpful in gaining justification for the system, as corporate officers see what is being purchased.

\subsubsection{Weaknesses}


The most obvious weakness is the potential to create an invalid model. The validity is at risk in this phase primarily because of the lack of historical data. This historical data forms the foundation for distribution selection, model verification, as well as giving a solid understanding of how the system is operated. Without this historical data, the simulation analyst is forced to rely on data from other similar systems, or from best guesses when no comparable system exists.

\subsubsection{Opportunities}

During the design phase the system has the greatest potential to take advantage of results of the simulation model. This is because the cost to revise the system does not involve changes to existing steel and concrete. Recommendations for system changes can readily be incorporated into the evolving design. Using the model to effect the evolution of the system provides the greatest probability of creating a system that will meet the needs of the end user both now and in the future.

\subsubsection{Threats}

The greatest threat to the success of the system in this phase is the caused by the chief weakness of the model. This is the threat of constructing a system based on results from an unverified, or invalid model. Aside from the lack of historical data, one of the chief causes of this threat is the lack of communication between the system supplier, and the end user. It is vital that the end user have a good understanding of their material handling needs. Without a good understanding, the material handling suppliers are left to define the needs as they perceive them. One of the common unpleasant experiences of end users is to inaccurately assume that the acceptance test created for the system is representative of how the system will function on an operational, day to day basis. This assumption is usually the result of the end user being unfamiliar with what they really need. There are often vast differences between what a system is designed to accomplish, and what it ends up being used for.

\subsection{Construction Phase}

The construction phase is defined as the time when the design is firm and contracts have been let for construction. Project teams have been created, and the design is defined on a micro basis.

\subsubsection{Strengths}

The model can be used as a reference point for all operational specifications that pertain to the tasks of the system. This reference point keeps the system in line with the design. Without the model as a reference, it is easy for the construction of the system to deviate from the proven design. When unexpected system revisions are discovered during construction, the model can be used to determine their consequences. If the consequences prove to be catastrophic to the system, the model can be used to develop recommendations to minimize their detriment.

\subsubsection{Weaknesses}

Because the design is firmly committed with the creation of contracts, and sub-contracts, the model has little ability to effect a physical change in the design of the system. Revisions to the model or uses of it during construction are usually rare except to study unexpected changes in the system.

\subsubsection{Opportunities}

During construction, there are few opportunities for system improvement. The "die is cast" so to speak and further evaluation must await the completion of construction. The only real opportunity is the minimization of the adverse effects of unexpected mandatory system revisions.

\subsubsection{Threats}

A significant threat exists to the system if the simulation model, and its results are abandoned. It is tempting to think that a small change to the system will not effect the performance of the system, and therefore progress without re-simulating the change. However, there are often unseen consequences which pose a serious threat to the success of the system. For this reason, the simulation effort must be updated for each change to the system.

\subsection{Service Phase}

After the system has evolved to a use that has proven consistent over time, the model can be updated to reflect the system "as-used". We have termed this phase the Service Phase.

\subsubsection{Strengths}

The strength of the model is its ability to more accurately predict the results of changes to the system. This increase in accuracy can be attributed to an increase 
in the availability of historical data. The incorporation of this data into the development of the model decreases the probability of relying on results from an invalid model. With a valid base model to work from, an almost infinite number of "what-if" scenarios can be simulated with extreme accuracy.

\subsubsection{Weaknesses}

The weakness of the model in this phase is its inability to effect change because of cost. Unlike the design phase where revision to steel and concrete are not an issue, the ability of the simulation analyst to make radical changes in the service phase is severely crippled. It is worth noting however, that this weakness will diminish with the passage of time. Inevitably, the needs of the user continue to evolve, and technology offers increasing return on investment for significant system revision.

\subsubsection{Opportunities}

Because of the abundance of historical data, and the subsequent creation of a valid model, opportunities to discover system performance improvements exist. Through the results of an appropriate design of experiments, significant improvements in system performance can be realized by implementing the findings from the simulation effort.

In addition to the more traditional formal simulation studies performed by consultants and material handling suppliers, companies are increasingly finding opportunities to utilize the simulation models themselves. The passage of a valid model to a user from a consultant or supplier allows the user to model on an almost infinite basis. Many users are finding that simulation can help them predict results on a daily basis. For example, a user could run the simulation model to determine the best sequence to create and ship orders of product. Using the simulation model in this way can create significant economies of scale with respect to system resource usage.

\subsubsection{Threats}

Like the design phase threat, the threat of implementing changes to steel and concrete based on the results of an invalid model is a significant threat to the system. Similar to this threat is the risk of not implementing changes which would have had a positive impact. This non-implementation is also the result of relying on invalid results.
Often a model can be validated against a particular "use" of the system only to be invalidated for another use. It is critical that the simulation effort be complete in its coverage of all distinct "uses" of the system. Without complete coverage, the risk of implementing changes to help one use of the system could be detrimental to another use.

\section{SUMMARY}

Understanding the evolution of an automated material handling model enables the simulation analyst to plan for the future and realize economies with respect to each phase of the model. For example, planning for the construction phase during the design phase, allows efficient passage of algorithms and data to software engineers as well as system information to project engineers.

The intent of this paper is to provide both end users of simulation results and simulation analysts with a framework for model evolution analysis. It is hoped that further strategic planning with respect to the stages identified in this paper would occur in all fields of simulation. This will aid in the quest for continuous use of simulation throughout the life of a system.

\section{AUTHOR BIOGRAPHY}

TODD HUNTER is the Lead Simulation Engineer at Eaton-Kenway, Inc, a world-wide supplier of Automated Material Handling Systems. He received a B.S. in Industrial Engineering from the University of Utah, and an MBA from Westminster College in Salt Lake City, Utah. 